\title{
Physical Properties of SmS Metallic Phase at Low temperature
}

\author{
M.Ohashi ${ }^{A D}$, D.Kondo ${ }^{A}$, N.Sato, T.Suzuki ${ }^{A}$, T.Komatsubara ${ }^{B}$, H.Takahashi ${ }^{C}$, and N.Môri ${ }^{\mathrm{D}}$ \\ Physics Department, ${ }^{A}$ Graduate School of Science, and ${ }^{B}$ Center for Low Temperature Science, \\ Tohoku University, Sendai 980-77,Japan \\ ${ }^{c}$ College of Humanities and Sciences,Nihon University,Tokyo 156,Japan \\ DISSP,University of Tokyo,Tokyo 106,Japan
}

\begin{abstract}
The resistivity of SmS single crystal has been measured under pressure up to $8 \mathrm{GPa}$. As an applied pressure is increased, the electrical resistivity changes drastically. In particular it shows a kink at $T_{M}$ in the $\rho$ vs $T$ curve above $2 \mathrm{GPa}$. We found that the electrical resistivity shows the $\rho=\rho_{0}+A T^{2}$ dependence with a large coefficient $A$ below $T_{M}$. If we ascribe this term to the electron-electron scattering, the electron correlation effect plays a dominant role in the high pressure region, which may correspond to the large $\gamma$ value.
\end{abstract}

[ SmS, resistivity, electron correlation, intermediate valent state, semiconductor-metal transition ]

\section{Introduction}

The compound SmS has been studied by a large variety of experimental methods. Application of high pressures induces the well known semiconductor-metal transition. The magnetic susceptibility of the collapsed phase of SmS lead Maple and Wohlleben[1] to the conclusion that $\mathrm{SmS}$ in the metallic phase, like $\mathrm{SmB}_{6}$ at atmospheric pressure, could be described by a system of ions showing intermediate valent state. The heat capacity measurements were reported to have a large $\mathrm{T}$-liner term coefficient, $\gamma=145 \mathrm{~mJ} / \mathrm{molK}^{2}$ at almost $1.5 \mathrm{GPa}[2]$. If we assume that it is due to the conduction-electron contribution, this may be an evidence of a strong electron correlation effect. Although many investigations have been performed so far, it may not be clearyet whether the electron electron correlation effect plays a dominant role in the metallic phase. Thus we have made the electrical resistivity measurements to study the electron correlation effect in $\mathrm{SmS}$ under high pressure.

\section{Experimental}

We prepared single crystals of $\mathrm{SmS}$ by the bridgman method in a sealed tungsten tube, under high vacuum. X-ray powder photographs confirmed both the $\mathrm{NaCl}$ structure and the lattice constant (5.925 $\AA$ ) corresponding to the earlier data of SmS (5.97 $\AA$ )[3]. At ambient pressure, the electrical resistivity is $28 \mathrm{~m} \Omega \mathrm{cm}$ at room temperature, and increases down to about $25 \mathrm{~K}$ by 260,000 times, below which the electrical resistivity was too large to be measured.

The resistivity of $\mathrm{SmS}$ has been measured up to $8 \mathrm{GPa}$. The sample of millimeter size is set up in a teflon cell containing a 1:1 mixture of fluorinate FC-70 and FC-77. The electrical contacts are Au-paste and Au-wire. Pressures have been applied using piston-cylinder $(\mathrm{p}<1.5 \mathrm{GPa}, 1.5 \mathrm{~K}<\mathrm{T}<300 \mathrm{~K})$ and cubic anvil press ( $\mathrm{p}>1.5 \mathrm{GPa}, 2.5 \mathrm{~K}<\mathrm{T}<300 \mathrm{~K}$ )[4], in which pressure load is kept constant during the measurements.

\section{Results}

Fig.1. represents the data at $p<1.2 \mathrm{GPa}$. Below room temperature the activation energy is estimated to be about 100 $\mathrm{meV}$ at ambient pressure when using a plot with $\rho=\rho_{0} \exp (-$ $\left.\Delta / 2 \mathrm{k}_{\mathrm{B}} \mathrm{T}\right)$.

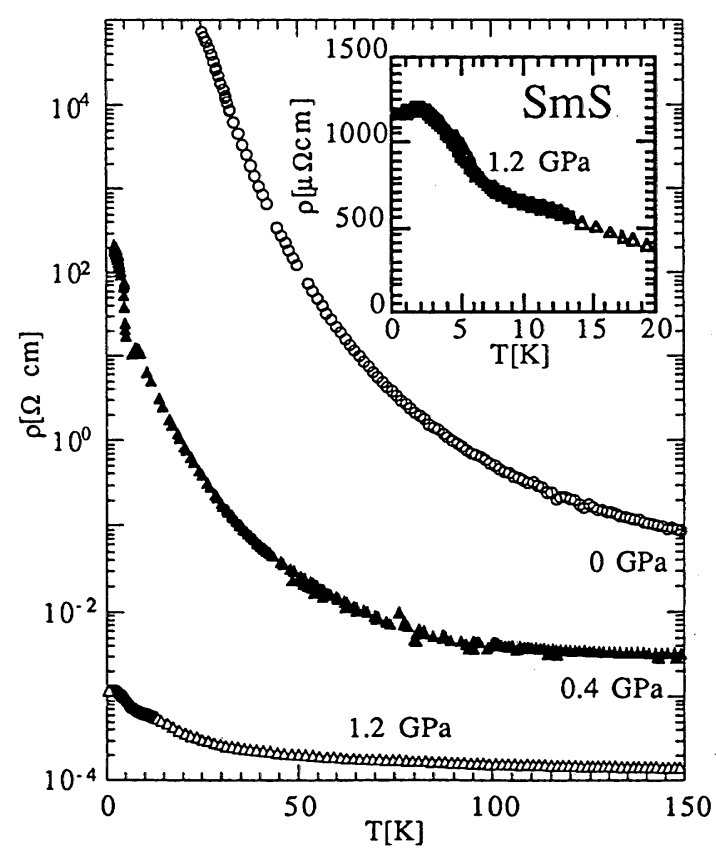

Fig.1. $\rho$ vs T curve at $p<1.2 \mathrm{GPa}$.

Fig.2. shows the electrical resistivity as a function of pressure is increased at room temperature. From Figs.1. and 2. it is clear that the transition is not a semiconductor-metal transition but a 
semiconductor-semiconductor transition takes place discontinuously at about $0.68 \mathrm{GPa}$. In addition, under $1.2 \mathrm{GPa}$, a small hump is observed on the $\rho$ vs T curve at about $13 \mathrm{~K}$, and the $\rho$ increases with decreasing temperature down to the temperature where the hump takesplace with $\Delta=4.5 \mathrm{meV}$ (Fig.1.). For $\mathrm{T}<2$ $\mathrm{K}$, however, the $\rho$ is found to decrease with decreasing temperature, while the resistivity at $0.5 \mathrm{~K}$ is found to be a very large value of $1180 \mu \Omega \mathrm{cm}$.

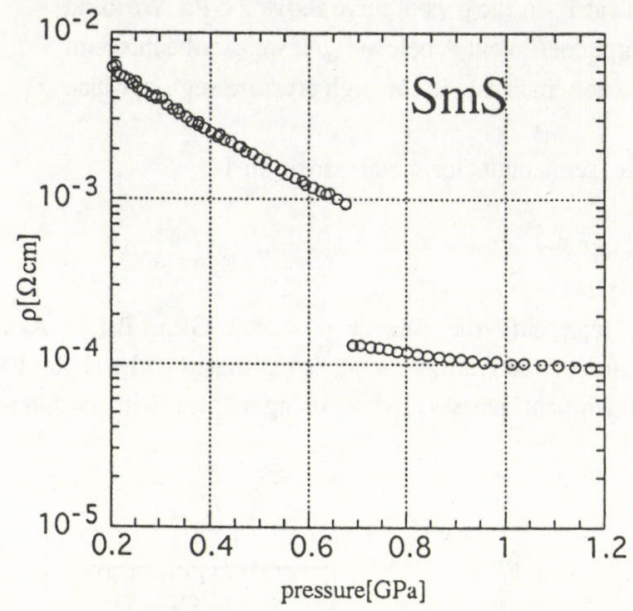

Fig.2. Electrical resistivity as a function of increasing pressure at room temperature.

As an applied pressure is increased furthermore, the electrical resistivity changes drastically as seen in Fig.3. Above $1.5 \mathrm{GPa}$, the electrical resistivity increases with decreasing temperature, while it does not obey an activation law. Moreover, an anomaly at $\mathrm{T}_{\mathrm{M}}$ appears above $2 \mathrm{GPa}$. $\mathrm{T}_{\mathrm{M}}$ is plotted as a function of pressure in fig.4.

\section{Discussion}

The new result is that a kink at $T_{M}$ under pressure in the $\rho$ vs $T$ curve is observed systematically, which is not shown in recent measurements[2][5]. Fig.4. shows the $T_{M}$ vs pressure curve, which suggests the presence of a magnetic ordering phase. However, no evidence of a magnetic ordering temperature has been reported so far.

It is evidenced that the electrical resistivity shows the $\rho=\rho_{0}+A T^{2}$ dependence under pressure more than $2 \mathrm{GPa}$ with a large coefficient $A$ below $T_{M}$. Fig.5. shows $\rho$ as a function of $\mathrm{T}^{2}$. $\rho_{0}$ and the coefficient $A$ rapidly decreases with increasing pressure as seen in Fig.6. A large coefficient A seems to reflect a strong electron correlation effect. The large $\gamma$ value was found in heat capacity at

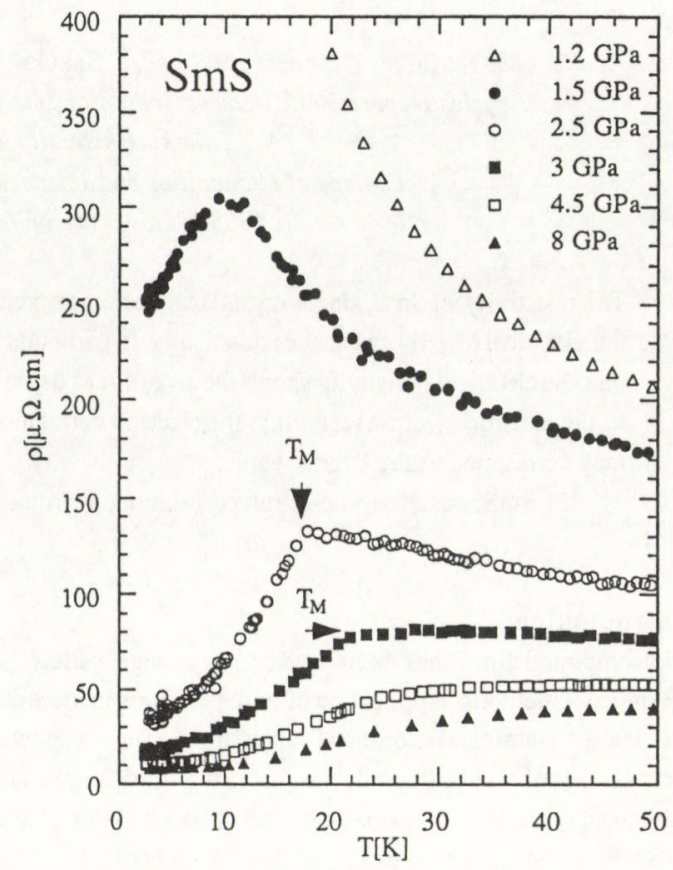

Fig.3. $\rho$ vs T curve at various pressure.

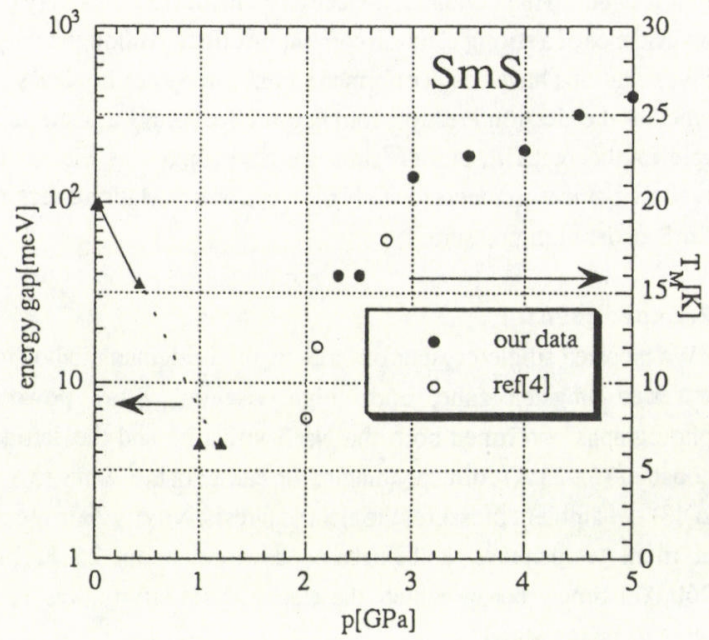

Fig.4. $T_{M}$ (circles), and activation energy (triangles) as a function of pressure.

1.5 GPa [1].If we assume that both $\mathrm{A}$ and $\gamma$ are caused by the same origin, the large $\gamma$ suggests the coefficient $A$ to be $0.2 \mu \Omega \mathrm{cm} / \mathrm{K}^{2}$ 
under 1.5 GPa [6], which seems to correspond well to the value obtained by an extrapolation in this study. However, it is difficult to find whether electrical resistivity shows $\rho=\rho_{0}+\mathrm{AT}^{2}$ dependence under $1.5 \mathrm{GPa}$ because of the pressure near the phase transition boundary. Moreover, the recent electrical resistivity and Hall coefficient measurements [5][7] suggested the existence of "Semiconducting Intermediate Valence State" at $\mathrm{p}<2 \mathrm{GPa}$. In order to make clear this point, the heat capacity in the metallic state and the pressure dependence of the $\gamma$ value should be measured.

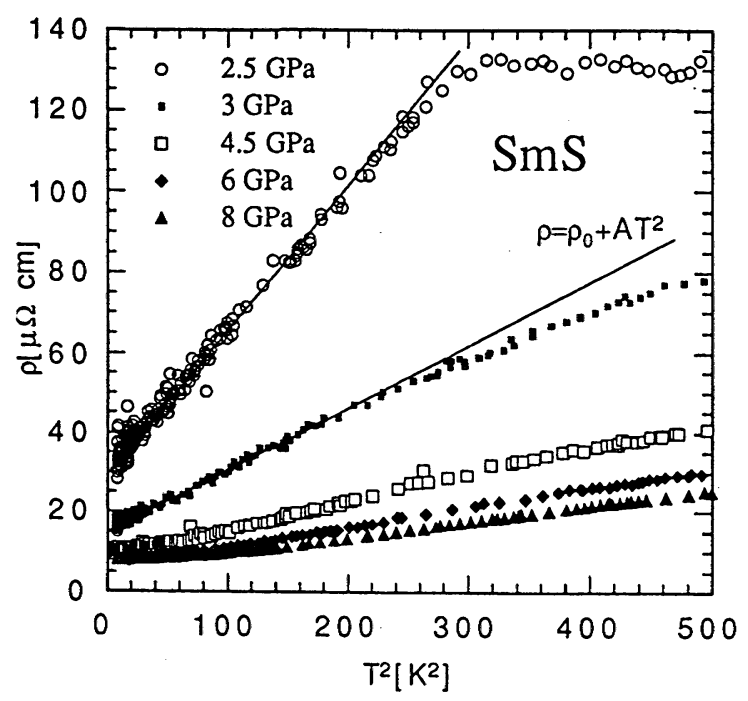

Fig.5. $\rho$ vs $\mathrm{T}^{2}$ curve under high pressure.

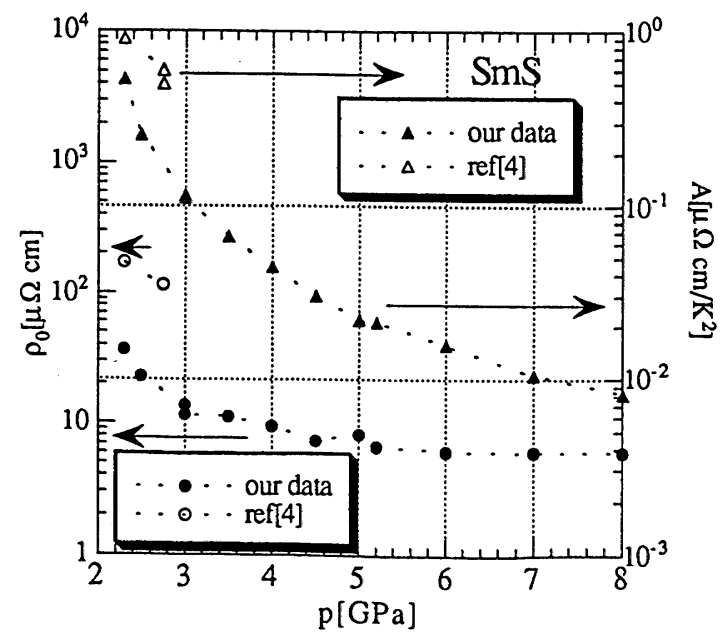

Fig.6. The pressure dependence on the $\rho_{0}$ (circles) and $A$ (triangles).

\section{References}

[1] M.B. Maple and D. Wohlleven, Phys. Rev. Lett., 27 511(1971)

[2] S.D. Bader, N.E. Phillips and D.B. McWhan, Phys. Rev. B., 7 4686(1973)

[3] R. Keller, G. Güntherodt, W.B. Holzapfel, M. Dietrich, F. Holtzberg, Solid State Commun., 29 753(1979)

[4] N.Mori, Y.Okayama, H.Takahashi, Y.Haga and T.Suzuki, Jpn. J. Appl. Phys. Series 8 182(1993)

[5] F. Lapierre, M. Ribault, F. Holtzberg and J. Flouquet, Solid State Commun., 40 347(1981)

[6] K. Kadowaki and S.B. Woods, Solid State Commun., 58 507(1986)

[7] M. Konczykowski, F. Lapierre,P. Haen, J. Morillo, and J.P. Senateur, J. Magn. Magn. Mat., $47 \& 48$ 277(1985) 